\title{
Navigation Modes for Combined Table/Screen 3D Scene Rendering
}

\author{
Rami Ajaj, Frédéric Vernier, Christian Jacquemin \\ LIMSI-CNRS and University Paris-Sud 11 \\ B.P. 133, 91403, Orsay Cedex, France \\ \{rami.ajaj, frederic.vernier, christian.jacquemin $\} @ 1 i m s i . f r$
}

\begin{abstract}
This paper compares two navigation techniques for settings that combine a 2D table-top view and a 3D large wall display, both rendering the same 3D virtual scene. The two navigation techniques, called Camera Based (CB) and View Based (VB) strongly rely on the spatial relationships between both displays. In the $\mathrm{CB}$ technique, the 3D point of view displayed on the wall is controlled through a draggable icon on the 2D table-top view. The VB technique presents the same icon on the table-top view but statically located at the center and oriented toward the physical wall display while the user pans and rotates the whole scene around the icon. While CB offers a more consistent 2D view, VB reduces the user's mental rotations required to understand the relations between both views. We perform a comparative user study showing user's preference for VB technique while performances for complex tasks are better with the CB technique. Finally we discuss other aspects of such navigation techniques, such as the possibility of having more than one point of view, occlusion, and multiple users.
\end{abstract}

\section{Keywords}

Interactive table-top, virtual reality, navigation

\section{INTRODUCTION}

In 3D User Interfaces, selection and manipulation of virtual objects is separated from navigation "which is the movement in and around the environment" [4, p. 136]. Interaction techniques are studied for navigation in 3D virtual environments in order to facilitate users input interactions as well as visualization. In the Virtual Reality field, several input devices are developed for virtual object manipulation and navigation in $3 \mathrm{D}$ virtual environments. These input devices usually integrate several $(3,6$ or even 12 degrees of freedom) in order to meet tasks requirements (e.g. 3D positioning, rotation and dimensioning of virtual objects). Depending on the input device, each has its advantages and its drawbacks but none fulfills all requirements of a 3D input device [19] such as ease of learning, speed, and fatigue avoidance.

There are numerous benefits of direct-touch table-top devices such as collaboration and natural data manipulation [15] because of input/output co-localization. Multi-touch interaction techniques preserve the input/output colocalization, enhance the functionality, and allow intuitive manipulation. However, planar manipulations on table-top direct-touch devices are not well-suited for 3D interactions because of the lack of degrees of freedom (DOF). Some interaction techniques are developed to cover 5 to 6 DOFs [11] but they provide only support for virtual object manipulation tasks.
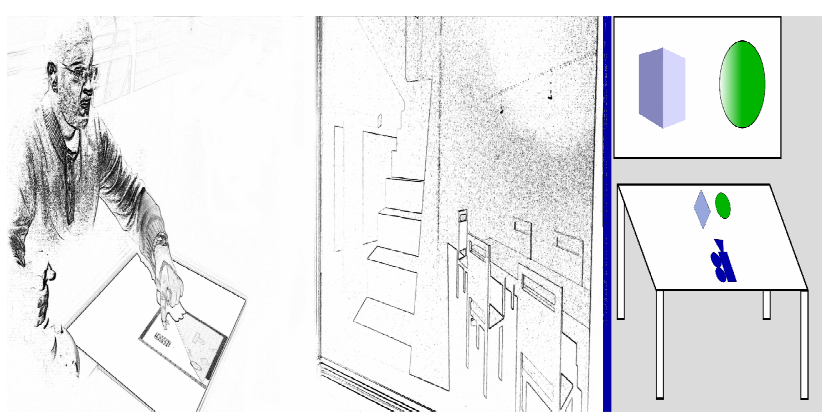

Figure 1: Left: A user interacting through the table-top device and using a joystick. Middle: The 3D corresponding view displayed vertically. Right: Overall interface. A 2D view and a camera icon projected on the table-top device and a 3D view on a vertical display.

In order to take advantage of the intuitive aspect of directtouch table-top devices for virtual object manipulation and navigation tasks in $3 \mathrm{D}$ virtual environments, we combine a table-top device with a vertical display surface and 3D input devices (see Figure 1). A 2D (map-kind) view of the virtual environment is built from the 3D model and projected on the table-top device. A 3D perspective view of the same virtual environment is shown on the vertical display. Our multimodal interface improves upon existing 3D interaction techniques by offering complementary, fast, and planar interactions. Essential elements such as existing relation between dimensions of both views must be considered when performing such combinations. Therefore, we design and compare two navigation techniques: in the first one users manipulate a camera icon displayed on the 
$2 \mathrm{D}$ view whereas in the second one, this camera icon is always pointing toward the physical vertical display and users manipulate the whole $2 \mathrm{D}$ view around it. In this paper we focus on horizontal navigation only. The contributions of this paper are the development and the comparison of these navigation techniques. The difference between these two techniques can be correlated with the usage of egocentric and world reference maps [5]. We compare them through a user study and a discuss occlusion, multiple cameras, and multiple users issues for these navigation techniques.

We first present related works and existing interfaces combining a table-top device with a companion vertical display. We also present existing navigation techniques in these interfaces. Then we describe the two interaction techniques and the comparative user study. Last, we compare these techniques for occlusion, multiple cameras, and multiple users situations.

\section{RELATED WORK}

The combination of a table-top device with a companion vertical display is studied for various applications and different table-top interfaces. Build-it [7] is a graspable planning tool combining a 2D planar view of a virtual environment displayed on a table-top device and a perspective $3 \mathrm{D}$ view projected on a wall. It is applied to architectural virtual environments such as a room. NAVRNA [3] is also a graspable table-top device combined with a vertical display but is specific to RNA (ribonucleic acid) molecule visualization, exploration, and edition. A 2D view called secondary structure is displayed on the table-top device whereas a 3D tertiary structure of the molecule is displayed on the vertical display. In Build-it and NAVRNA, users interact using physical props (bricks for Build-it and tokens for NAVRNA) on the table-top device. Aliakseyeu et al. [2] develop a setup combining also a $2 \mathrm{D}$ view projected on a table-top device and a $3 \mathrm{D}$ complementary view displayed on a vertical screen. Users manipulate a physical prop (in a form of a wired frame) on top of the table-top device in order to visualize slices of the virtual object as if it was located in this area. The realworld layout of the two views is not studied in all these research works.

For direct-touch table-top devices, Forlines et al. [10] combine a $2 \mathrm{D}$ top-down view displayed on a table-top device with $3 \mathrm{D}$ views on vertical screens for a geospatial application. Interactions are performed through the tabletop device and a complementary tablet PC. A title bar on the table-top $2 \mathrm{D}$ view is also displayed on vertical views in order to facilitate user's understanding of actual relationships between both views. However, feedback about real world relationship between views is not displayed. Wigdor et al. [18] combine a table-top device with multiple vertical displays also. The relationships are highlighted by multiple patterns such as colors and visual connectivity between displays. This system offers a feedback about physical relationships between different views to users. However, it is not applied to a virtual environment and does not manage navigation.

Wigdor et al. [17] study the effect of the position of the vertical display with respect to the table-top device and orientation of the control space on the table-top device on user's preference and performance. Results show that user's preference is correlated to her/his comfort rather than to her/his performance. Results also show that users slightly orient the control space in the direction of the vertical screen. Last, the vertical display position behind the user is inappropriate. This work highlights the importance of the vertical display orientation with respect to the table-top device.

Fjeld et al. [8] [9] develop the GroundCatcher and the FrameCatcher techniques for navigation in a $2 \mathrm{D}$ view projected on a table-top device within the Build-it planning tool [7]. In GroundCatcher, users manipulate the whole 2D view whereas in FrameCatcher, users manipulate a frame displayed on the 2D view and at deselection, the frame becomes the view port of the $2 \mathrm{D}$ view. They also develop the Camera and Window techniques for the navigation in the $3 \mathrm{D}$ view displayed on the wall. In the Camera technique users manipulate a Camera that represents the 3D point of view, whereas in the Window technique, users manipulate a window that represents the $3 \mathrm{D}$ view. In another work, Fjeld et al. [6] develop the Camera technique by adding active objects in order to handle 3D rotations and zoom.

Kuchar et al. [13] work on visualizing a cultural heritage by displaying a 2D top-down view on a touch screen and a 3D view on a wall in front of the touch screen. A representation of the $3 \mathrm{D}$ viewpoint on the $2 \mathrm{D}$ view is surrounded by four equally separated areas. Navigation is performed by selecting one of the four areas (e.g. in front to move forwards) and speed is related to the distance between the $3 \mathrm{D}$ viewpoint representation and the touch position. All these studies do not handle existing physical relationships between both views. As Wigdor et al. [17] demonstrate, this issue is crucial to facilitate users' understanding of existing relations between views and to simplify the focus switch between both views. We develop the Camera-Based (CB) navigation technique that is similar to the Camera technique and the View-Based (VB) technique that relies on real view relationships. Both techniques are compared through a user study.

\section{CAMERA-BASED NAVIGATION TECHNIQUE}

$\mathrm{CB}$ navigation technique is based on direct-touch planar manipulation of the $3 \mathrm{D}$ viewpoint representation in a $2 \mathrm{D}$ view (see Figure 2). The aim of this technique is to facilitate navigation in a virtual environment and to preserve the user's aerial view of the environment. In this technique, the user manipulates the camera icon in the $2 \mathrm{D}$ view. The modifications on the camera icon are immediately reflected on the viewpoint of the $3 \mathrm{D}$ view. This technique is similar to the Camera technique [8] [9] 


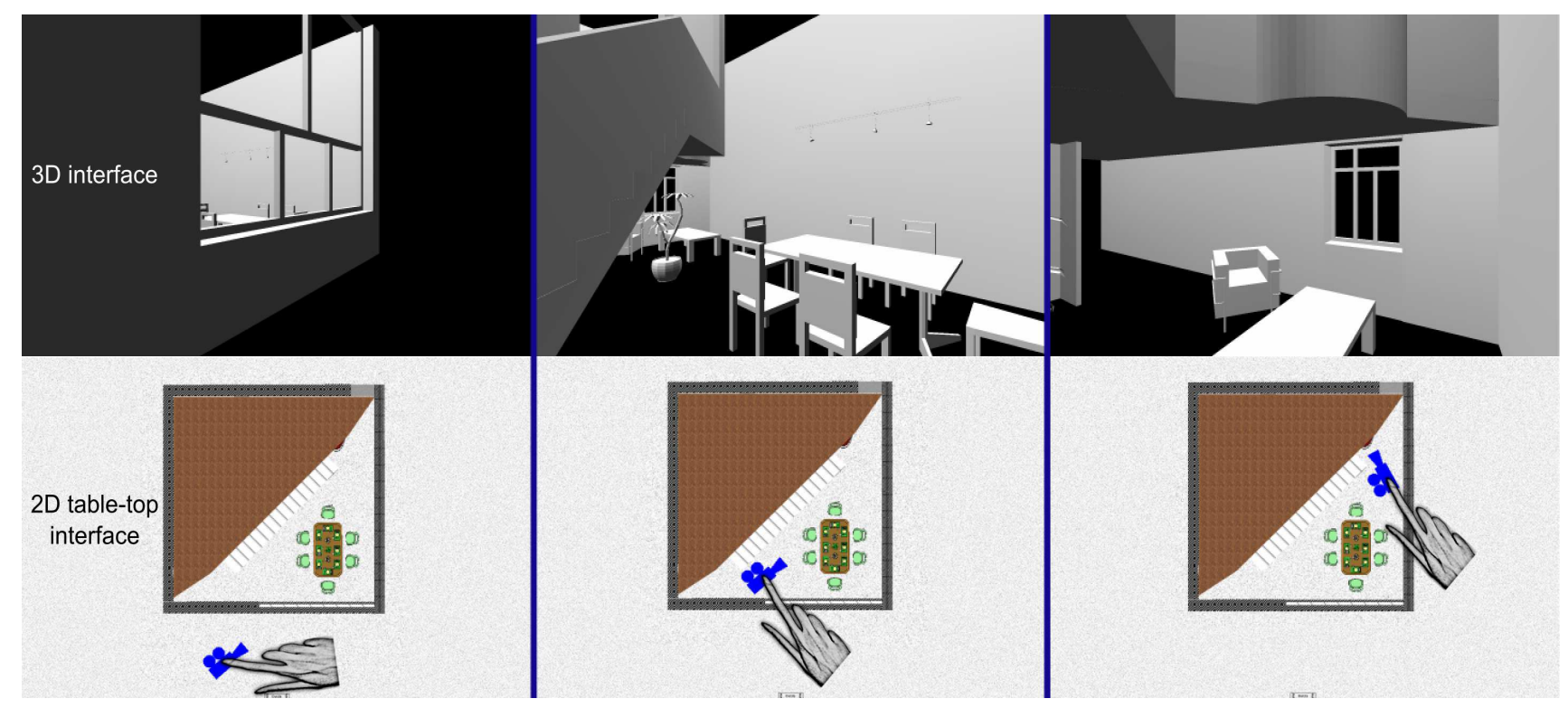

Figure 2: Camera-Based (CB) navigation technique. Bottom: 2D view and interaction. Three different camera icon positions in the 2D view manipulated by direct-touch and displayed on the table-top device. Top: 3D view and interaction. The 3D view is shown on a vertical display and corresponds to the $2 \mathrm{D}$ camera icon position given

and the eyeball in hand metaphor [16] but is applied to a direct-touch table-top device.

Since CB technique is based on direct manipulation through tactile interaction on a table-top interface, it can be easily extended to other interaction techniques developed for data manipulation on interactive surfaces. For instance we have applied the Rotate'N Translate technique [12] to the camera icon and this allows fluid 3 DOFs horizontal navigation [1]. Horizontal rotation can also be performed by manipulating a graphical widget attached to the camera icon or by physical finger orientation as in TNT techniques [14]. Two finger interaction techniques could also be applied for horizontal 3D point of view rotation but have not been developed so far.

In $\mathrm{CB}$, planar direct touch interactions for 3D viewpoint direct manipulation offer easy, intuitive, and fast horizontal navigation in a virtual environment. Another advantage of this technique is the fixed 2D view position and orientation that allow the user to have a consistent overview of the virtual scene at any time and the facility of its mental representation by the user.

During navigation, the camera icon orientation changes with respect to user's interactions. Therefore, the user must perform a mental rotation in order to understand the location of the $3 \mathrm{D}$ view (displayed vertically) in the virtual scene. Another drawback of the $\mathrm{CB}$ technique is the necessity of camera icon selection and manipulation. Large table-top devices make the use of this technique slightly uncomfortable. If the camera icon is physically far from the user, she/he must reach out toward it making the interaction possibly difficult to perform.

\section{VIEW-BASED NAVIGATION TECHNIQUE}

$\mathrm{VB}$ navigation technique facilitates navigation in a $3 \mathrm{D}$ virtual environment by planar manipulation of the whole $2 \mathrm{D}$ view. The aim of this technique is to help the user to quickly understand the spatial relationship between the two views displayed on distinct perpendicular surfaces. A coupling between the virtual views and the physical surfaces is made in the VB technique: the camera icon in the $2 \mathrm{D}$ view always points at the physical vertical surface (see Figure 3).

In $\mathrm{VB}$, a representation of the physical vertical surface is shown in the $2 \mathrm{D}$ view (black rectangle in upper part of Figure 3 bottom). The camera icon displayed in the center of the 2D view cannot be modified and always points at the vertical surface physical location in the room. Translations in the $3 \mathrm{D}$ view are performed by manipulating the whole $2 \mathrm{D}$ view. Horizontal rotations are performed by selecting a graphical button displayed on the $2 \mathrm{D}$ view and then manipulating the whole $2 \mathrm{D}$ view that rotates around the camera icon (representing the 3D point of view). Multitouch interactions have not been developed yet because of the necessity to perform rotations around a fixed point (i.e. the center of the camera icon) and in order to preserve input/output coupling that is essential for direct-touch tabletop interfaces [11].

The VB interaction technique for navigation is similar to the scene in hand metaphor developed by Ware and Osborne for 3D virtual environments [16]: in both techniques the user manipulates the virtual scene with respect to a fixed viewpoint. However, the scene in hand metaphor is used with a 6 DOF input device and is applied to a single view whereas interactions that use VB technique are planar and are applied to one view in order to navigate 


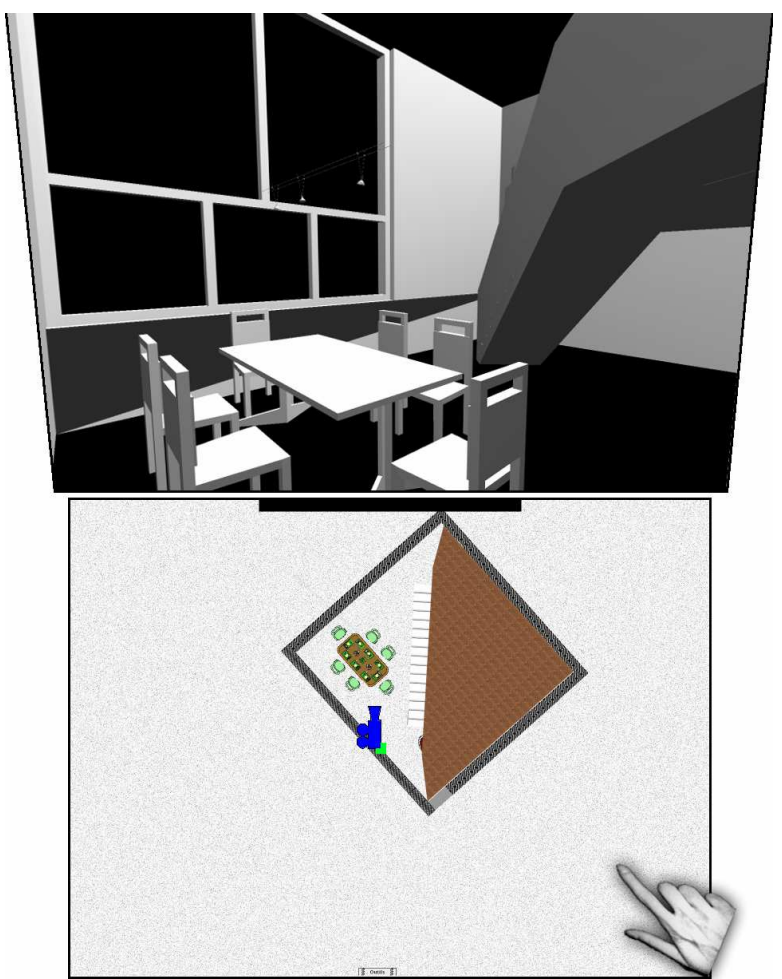

Figure 3 : View-Based (VB) navigation technique. Bottom: 2D view projected on the table-top device with the view-based navigation technique. Top: the 3D corresponding view shown on the vertical display.

through another one. In VB technique, the 2D camera icon and the representation of the vertical surface on the 2D view are fixed. The $2 \mathrm{D}$ view changes with respect to the $2 \mathrm{D}$ camera representation.

The advantages of the VB navigation technique is the fixed camera icon orientation that is always pointing at the physical vertical surface. Because the user, the camera icon, the screen black icon, and the vertical surface are aligned, she/he does not have not perform a mental rotation in order to understand the $3 \mathrm{D}$ view. The second advantage of VB is the large selection area for navigation. The last advantage is the constant and large display area on the table-top device dedicated to the rear of the camera missing in the $3 \mathrm{D}$ view.

The main VB technique drawback is the view displacement with respect to the table-top device. This displacement may prevent the user from keeping a consistent and coherent mental representation of the virtual environment. Therefore the user might get lost more easily when compared with a fixed $2 \mathrm{D}$ overview.

\section{USER STUDY}

We describe a user study to evaluate the respective merits of $\mathrm{CB}$ and $\mathrm{VB}$ techniques.

\section{Goals}

The aim of the user study is to compare user performances and preferences between $\mathrm{CB}$ and VB navigation techniques.

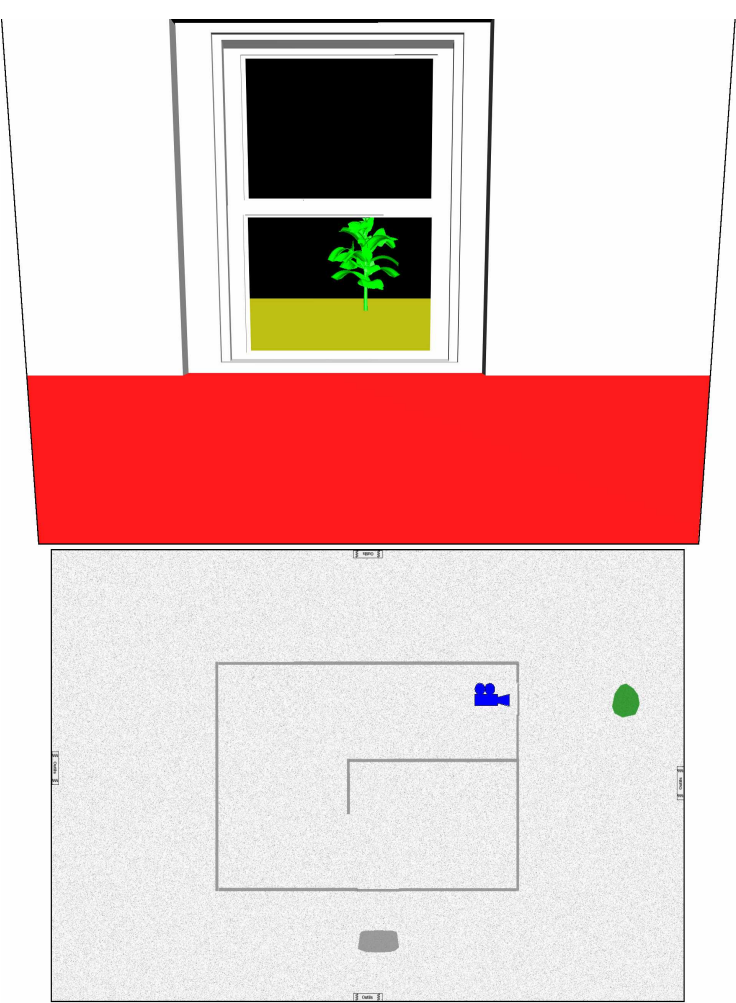

Figure $4: 2 D$ and $3 D$ views of the virtual environment for the tree task using the CB navigation technique.

Darken and Cevik [5] observe that performance between egocentric and world-reference map usage depends on task type. In our study, CB is similar to the world-reference map whereas VB is similar to the egocentric map. Therefore, based on Darken and Cevik observations, we hypothesize that:

- Performance and user preference should be better using VB technique for egocentric tasks (e.g. targeted search task).

- $\quad$ Performance and user preference should be better using CB technique for exocentric tasks (e.g. naïve search task).

Hence, the goal of the user study is to test, validate or invalidate these two hypotheses.

\section{Procedure}

Two tasks are considered in the evaluation setup in order to measure user preference and performance and relate them to task type. In the first task, that we call tree task, users are situated in a virtual house with two windows (see Figure 4). Outside, in front of the first window there is a virtual car and in front of the second one there is a tree. Users are asked to position themselves in front of the second window in a perpendicular way and to have the best view on the tree outside through the window. In the second task, that we call red ball task, the virtual environment is composed of fifteen houses each containing two plants (see Figure 5). Somewhere a unique red ball exists in the virtual 


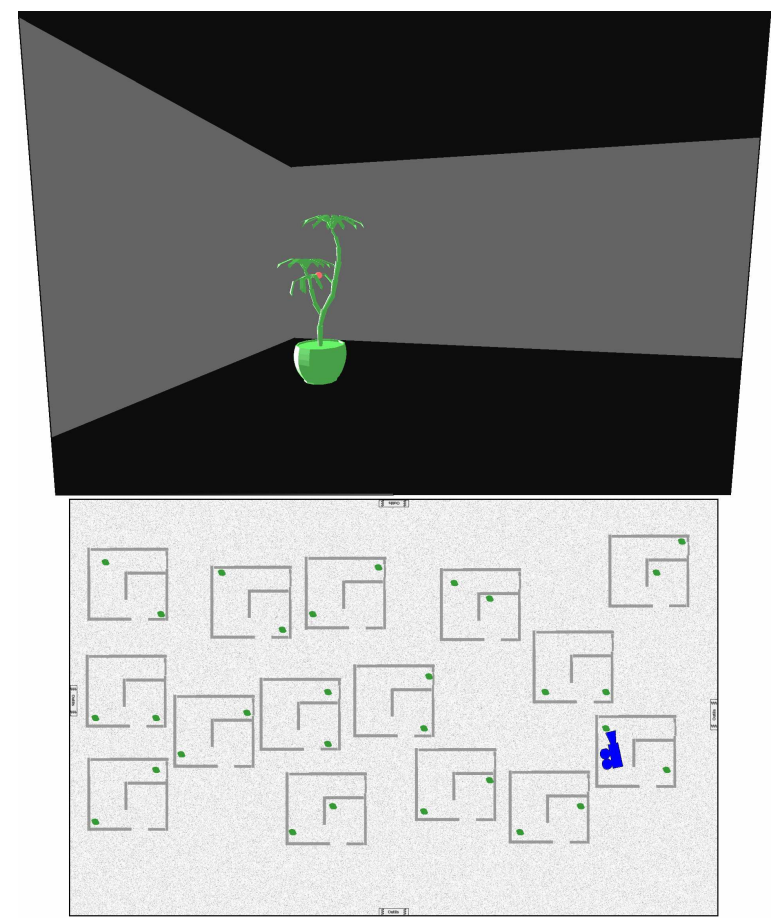

Figure 5 : 2D and 3D views of the virtual environment for the red ball task using the CB navigation technique. The red ball is visible only in the 3D.

environment next to one of the thirty plants. Users are asked to find the red ball that is displayed only in the $3 \mathrm{D}$ view. The tree task is an egocentric task because the absolute position and orientation of the 3D viewpoint is not as important as the relative position and orientation with respect to a virtual object (here a virtual window). The red ball task requires users to remember which plants they have already viewed in order to avoid revisiting them (to find the red ball) and therefore a good knowledge of their own position in the world reference of the virtual environment is essential.

We use the Management Cabin (MC) setup [1] to perform our user study. In MC, a 2D (map-kind) view of a virtual scene is displayed on a table-top device as well as a camera icon that represents the viewpoint on the $3 \mathrm{D}$ view displayed on a companion vertical screen. Since rotation cannot be set with the same technique in $\mathrm{CB}$ and in $\mathrm{VB}$, and since $\mathrm{MC}$ offers multiple input device combination, we choose to add a joystick to perform the same rotations in the user study. Hence, translations are performed using the table-top device only and rotations using the joystick only.

24 users (10 females and 14 males) with various education backgrounds and experience levels in 3D virtual environment and 3D games participated in the evaluation. At the beginning of the experiment, each user had five minutes to test freely both navigation techniques. Afterwards, users are asked to perform each task with each navigation technique four times with different final positions each (different final window position for the tree task and different final red ball position for the red ball task). The four trials allow to highlight the random aspect of red ball positioning in the virtual scene. Task order and navigation technique order were counter-balanced. Users indicate freely when the task is accomplished. The experiment lasts approximately 30 minutes.

During and at the end of the evaluation, users are asked to answer a series of questions in order to obtain a subjective comparison between both navigation techniques. Users must rate after performing each task with one of the navigation techniques the ease, satisfaction, intuitivity, and efficiency of interactions. Ratings are realized on a scale of 7 where 1 is totally negative, 4 is neutral, and 7 totally positive. After performing each task with both navigation techniques, users must choose their preferred navigation technique for the given task. The same question is asked about the overall navigation technique preference at the end of the evaluation.

\section{Results and Analysis}

Objective data for completion time average is acquired during evaluation. Average navigation time for each task and with each navigation technique is computed with the three best times performed by each user. Time results for tree task (see Figure 6) show no significant difference between both navigation techniques $(\mathrm{t}(46)=0.17, \mathrm{p}=0.863)$. Average time is similar as well as the standard deviation which is slightly higher for the $\mathrm{CB}$ technique. Hence, our first hypothesis is not verified.

Average time results for the red ball task (see Figure 7) show better time performance for $\mathrm{CB}$ technique than for VB technique $(\mathrm{t}(46)=-2.37, \mathrm{p}=0.022)$. Some users highlight that they get lost in the virtual scene while navigating with the VB technique and could not remember which plants they have already visited previously. Hence, they might have revisited some plants and it results in a higher average time (in comparison with $\mathrm{CB}$ ). These results sustain our second hypothesis where user performance is better using the $\mathrm{CB}$ technique for exocentric tasks.

Users' ratings of ease, satisfaction, intuitivity, and

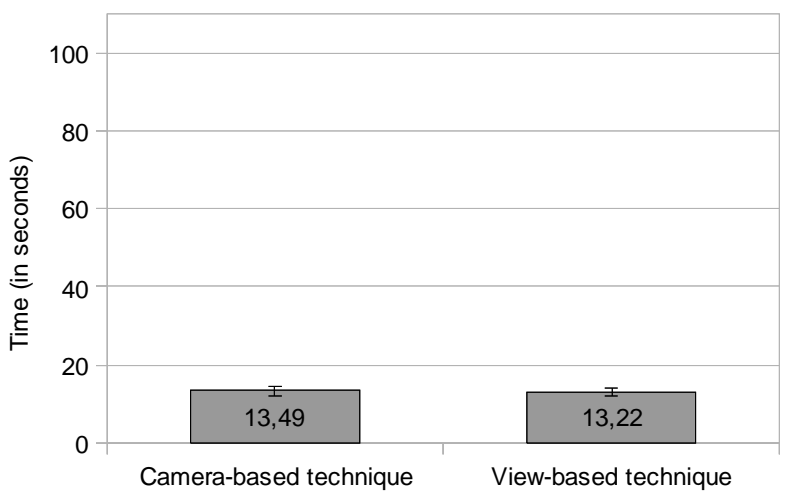

Figure 6 : Average time results in seconds needed to accomplish tree task. 


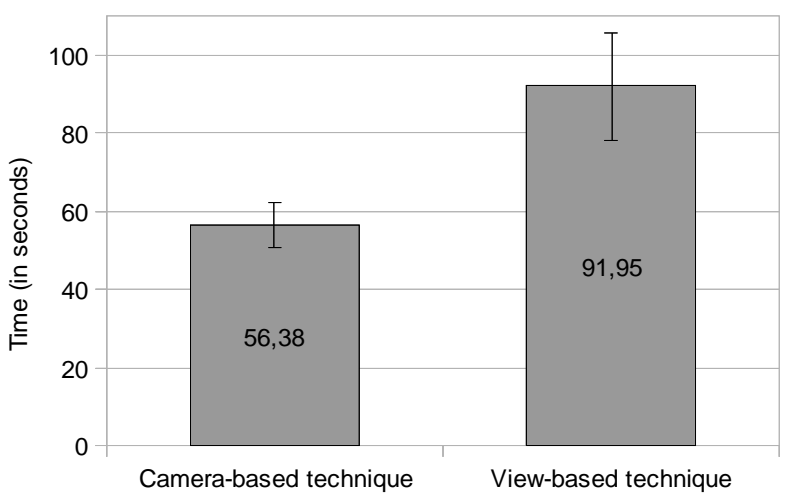

Figure 7 : Average time results in seconds needed to accomnlish rod hall task.

efficiency (see figure 8) show slight differences but are not very significant. Nevertheless, users' preferences show that 73 percent (respectively 71 and 62 percent) of them prefer VB technique generally (respectively for tree task and red ball task). Users justify their choice by ease of interaction with VB because selection and manipulation can be performed at any place of the view and not at a specific place as for the $\mathrm{CB}$ technique. In $\mathrm{CB}$ technique, users interact with the camera icon whereas in VB users can select and manipulate any free point (i.e. where there is no virtual object and camera icon) of the $2 \mathrm{D}$ view to initiate a translation.

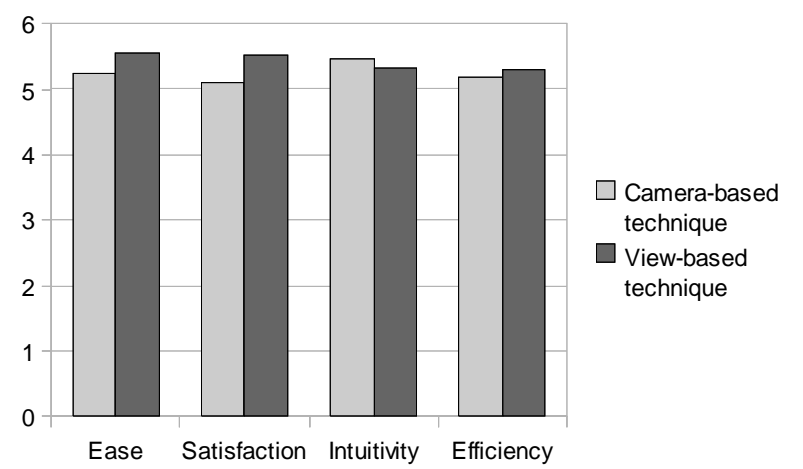

Figure 8 : Average of all users ratings of interaction for ease, satisfaction, intuitivity, and efficiency.

In summary, users prefer and rate generally better VB than $\mathrm{CB}$ technique. Even though users prefer VB technique, average time results show that time needed to accomplish the exocentric task is lower when using CB technique. The fact that some users get lost in the virtual scene when using the VB technique explains these time results that supports our second hypothesis shown by longer access times.

\section{DISCUSSION}

On the one hand, occlusion and multiple users are key issues when developing table-top interaction techniques. On the other hand, switching between several viewpoints is desired when visualizing complex 3D virtual scenes. We discuss here the $\mathrm{CB}$ and $\mathrm{VB}$ navigation techniques for these purposes.

\section{Occlusion}

In the $\mathrm{CB}$ technique, the $2 \mathrm{D}$ view is centered on a fixed position in the virtual environment. This allows the user to have a good overview of the virtual scene without occluding any part of it when a proper initial scaling and positioning is done. Therefore, for non complex environments, a zoom functionality is not useful for the 2D view. However, camera icon selection through direct-touch increases occlusion produced by user's hands [15].

The $2 \mathrm{D}$ view in the $\mathrm{VB}$ technique is centered on the camera icon (see View-based Navigation Technique section). The view position and orientation on the table-top device are modified by navigation and therefore parts of the virtual scene can get out of reach of the 2D view. Hence, a zoom functionality may be necessary for the $2 \mathrm{D}$ view in order to overcome this problem. Although occlusion occurs because of camera centered 2D view, physical occlusion produced by user's hands is reduced in the VB technique because of the large selection area (users start to pan at any free area of the view that does contain neither virtual objects nor a camera icon).

\section{Multiple Cameras}

Multiple camera icons representing different 3D viewpoints can be displayed on the $2 \mathrm{D}$ view. Only one camera icon, the $3 \mathrm{D}$ viewpoint of the vertical surface, can be active at a time. Inactive camera icons can be manipulated without altering the current 3D view which allows "offline" viewpoint positioning. Viewpoints can be easily changed from one camera icon to another by activating a button on the desired camera icon. This offers fast and easy switch between multiple predefined viewpoints.

The manipulation of one camera icon within the $C B$ technique does not affect simultaneous manipulation of other camera icons. The viewpoint teleportation is usually dissuaded in virtual environments in order to avoid user's disorientation. But since the $2 \mathrm{D}$ view does not change with respect to the table-top device, the user keeps always an overview of the virtual scene. Therefore, the viewpoint switch from an active camera to an inactive one can be done directly without the additional animation.

When VB technique is used, the whole $2 \mathrm{D}$ view is moved and rotated in order to navigate in the virtual environment. Hence, simultaneous navigation and positioning of an inactive camera icon in the virtual scene is difficult to perform. Nevertheless, simultaneous inactive cameras is possible without any inconvenience. Another important issue in VB technique usage is the switch from an active camera to an inactive one because of the camera centered $2 \mathrm{D}$ view. Indeed, when switching between inactive and active cameras, both views are modified and disorient users. An animation would help users to overcome disorientation when switching between two cameras. We recommend that this animation should interpolate the center of the $2 \mathrm{D}$ view and the $3 \mathrm{D}$ view from the current active camera icon to the newly activated one. 

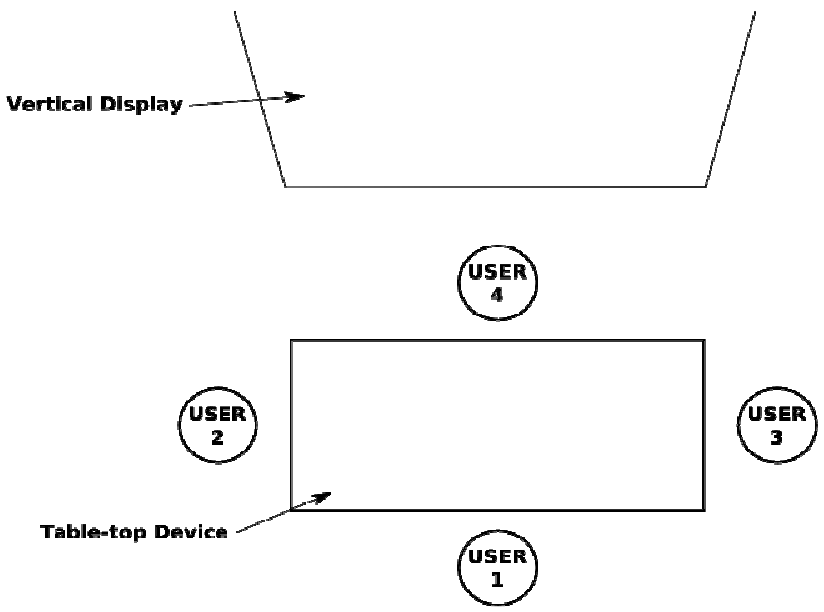

Figure 9 : Position of four users sitting around the table-top device relatively to the vertical display.

\section{Multiple Users}

An important benefit of table-top devices is that several users can sit around and share interactions simultaneously. Of course, each of them has a different view on the 2D and $3 \mathrm{D}$ views (see Figure 9). The user located in front of the table-top device and the vertical display (user 1 of Figure 9) is the most advantaged because views are aligned. Both users located perpendicularly to the vertical display when sitting in front of the table-top device (user 2 and user 3 of Figure 9) are equally situated. However, they are disadvantaged when compared to the user sitting directly in front of the vertical display because of the necessity of a 90 degrees horizontal head rotation in order to switch between both views. Last, the user turning her/his back to the vertical display (user 4 of Figure 9) is the most disadvantaged one because she/he must make a 180 degrees rotation to access the $3 \mathrm{D}$ view. For this user, the vertical display is almost unusable [17].

In the VB technique, the unfairness of user's position around the table-top device is even increased. A user sitting perpendicular (user 2 or user 3 of Figure 9) or turning her/his back (user 4 of Figure 9) to the vertical display is much more disadvantaged than the user sitting in front of the vertical display (user 1 of Figure 9). Actually the user sitting in front of the vertical display has the optimal position because of user, camera icon, and vertical view are aligned. This alignment eliminates the need for mental rotation to understand the relation between the $2 \mathrm{D}$ and $3 \mathrm{D}$ views.

For the $\mathrm{CB}$ technique, a fixed $2 \mathrm{D}$ view allows each user to have her/his own consistent mental representation of the virtual environment depending on her/his location around the table-top device. Therefore, $\mathrm{CB}$ technique reduces location unfairness around the table-top device. Moreover, if multiple vertical displays (aligned to different table-top borders) are used, multiple camera icons can be activated at the same time and thus reduce user's unfairness.

\section{CONCLUSION AND FUTURE WORK}

This paper has presented two interaction techniques for navigation in a virtual environment in a setup combining a 2D (map-kind) view displayed on a table-top device and a $3 \mathrm{D}$ view displayed on a companion vertical display. The CB technique is suitable for tasks where a mental representation of the virtual scene is needed because of a fix and consistent 2D view display. Moreover it reduces unfairness between multiple users sitting around the tabletop device. The VB technique has other advantages such as reducing mental rotation needed by users to understand the relation between both views and reducing occlusion produced by physical hands during interactions. Most of the users prefer this technique to CB. Nevertheless, in this technique users do not have a consistent overview of the virtual scene and therefore users might get disoriented. Moreover, when replacing the table-top device with a PDA or a tabletPC, the coupling between both views should be preserved in the $\mathrm{VB}$ technique (in $\mathrm{CB}$ it is not necessary).

The various advantages and drawbacks of $\mathrm{CB}$ and VB techniques suggest to combine them. This combination could be made by offering users the possibility to switch dynamically between the two navigation techniques. Another interesting idea is to test an automatic navigation technique switch at the end of user's interactions. The aim of this switch would be to help users quickly understand the relation between both views in the $\mathrm{VB}$ technique and to quickly locate her/his camera position in the virtual scene in the $\mathrm{CB}$ technique. A user experiment will be performed for users not directly located in front of the vertical screen. The aim of this experiment would be to compare between both navigation techniques the influence of user's position around the table-top device on her/his spatial perception. The design space for view manipulation is large which encourages to develop new techniques. Last, multiple vertical displays could be added to the table-top/vertical screen setup and an extension of the $\mathrm{CB}$ and VB navigation techniques could be performed to integrate these displays.

\section{REFERENCES}

1. Ajaj, R., Vernier, F., and Jacquemin, C. 2009. Follow My Finger Navigation. In Proceedings of HumanComputer Interaction INTERACT 2009 (Uppsala, Sweeden, August 24 - 28, 2009). Gross, T., Gulliksen, J., Kotzé, P., Oestreicher, L., Palanque, P., Prates, R.O., Winckler, M. Eds. Lecture Notes In Computer Science, vol. 5727. Springer-Verlag, Berlin, Heidelberg, 228231.

2. Aliakseyeu, D., Subramanian, S., Martens, J., and Rauterberg, M. 2002. Interaction techniques for navigation through and manipulation of 2D and 3D data. In Proceedings of the Workshop on Virtual Environments 2002 (Barcelona, Spain, May 30 - 31, 2002). W. Stürzlinger and S. Müller, Eds. ACM International Conference Proceeding Series, vol. 23. 
Eurographics Association, Aire-la-Ville, Switzerland, 179-188.

3. Bailly, G., Nigay, L., and Auber, D. 2006. NAVRNA: visualization - exploration - editing of RNA. In Proceedings of the Working Conference on Advanced Visual interfaces (Venezia, Italy, May 23 - 26, 2006). AVI '06. ACM, New York, NY, 504-507.

4. Bowman, D., Kruijff, E. LaViola, J. and Poupyrev, I., 2004. 3D User Interfaces: Theory and Practice. Addison-Wesley Professional.

5. Darken, R. P. and Cevik, H. 1999. Map Usage in Virtual Environments: Orientation Issues. In Proceedings of the IEEE Virtual Reality (March 13 - 17, 1999). VR. IEEE Computer Society, Washington, DC, 133-140.

6. Fjeld, M., Ironmonger, N., Voorhorst, F., Bichsel, M., Rauterberg, M. 1999. Camera control in a planar, graspable interface. In Proceedings of 17th IASTED AI'99, 242-245.

7. Fjeld, M., Lauche, K., Dierssen, S., Bichsel, M., and Rauterberg, M. 1998. BUILD-IT: A Brick-based integral Solution Supporting Multidisciplinary Design Tasks. In Proceedings of the IFIP Working Group 13.2 Conference on Designing Effective and Usable Multimedia Systems A. G. Sutcliffe, J. Ziegler, and P. Johnson, Eds. IFIP Conference Proceedings, vol. 133. Kluwer B.V., Deventer, The Netherlands, 58.

8. Fjeld, M., Voorhorst, F., Bichsel, M., and Krueger, H. 1999. Exploring brick-based camera control. In Proceedings of the HCI international '99 (the 8th international Conference on Human-Computer interaction) on Human-Computer interaction: Communication, Cooperation, and Application DesignVolume 2 - Volume 2 (August 22 - 26, 1999). H. Bullinger and J. Ziegler, Eds. L. Erlbaum Associates, Hillsdale, NJ, 1060-1064.

9. Fjeld, M., Voorhorst, F., Bichsel, M., Lauche, K., Rauterberg, M., and Krueger, H. 1999. Exploring BrickBased Navigation and Composition in an Augmented Reality. In Proceedings of the 1st international Symposium on Handheld and Ubiquitous Computing (Karlsruhe, Germany, September 27 - 29, 1999). H. Gellersen, Ed. Lecture Notes In Computer Science, vol. 1707. Springer-Verlag, London, 102-116.

10.Forlines, C., Esenther, A., Shen, C., Wigdor, D., and Ryall, K. 2006. Multi-user, multi-display interaction with a single-user, single-display geospatial application. In Proceedings of the 19th Annual ACM Symposium on User interface Software and Technology (Montreux, Switzerland, October 15 - 18, 2006). UIST '06. ACM, New York, NY, 273-276.
11.Hancock, M., Carpendale, S., and Cockburn, A. 2007. Shallow-depth $3 \mathrm{~d}$ interaction: design and evaluation of one-, two- and three-touch techniques. In Proceedings of the SIGCHI Conference on Human Factors in Computing Systems (San Jose, California, USA, April 28 - May 03, 2007). CHI '07. ACM, New York, NY, 1147-1156.

12. Kruger, R., Carpendale, S., Scott, S. D., and Tang, A. 2005. Fluid integration of rotation and translation. In Proceedings of the SIGCHI Conference on Human Factors in Computing Systems (Portland, Oregon, USA, April 02 - 07, 2005). CHI '05. ACM, New York, NY, 601-610.

13. Kuchar, R., Schairer, T., and Straßer, W. 2007. Photorealistic Real-time Visualization of Cultural Heritage: A Case Study of Friedrichsburg Castle in Germany. In Proceedings of EuroGraphics Cultural Heritage, 9-16.

14.Liu, J., Pinelle, D., Sallam, S., Subramanian, S., and Gutwin, C. 2006. TNT: improved rotation and translation on digital tables. In Proceedings of Graphics interface 2006 (Quebec, Canada, June 07 - 09, 2006). ACM International Conference Proceeding Series, vol. 137. Canadian Information Processing Society, Toronto, Ont., Canada, 25-32.

15.Shen, C., Ryall, K., Forlines, C., Esenther, A., Vernier, F. D., Everitt, K., Wu, M., Wigdor, D., Morris, M. R., Hancock, M., and Tse, E. 2006. Informing the Design of Direct-Touch Tabletops. IEEE Comput. Graph. Appl. 26, 5 (Sep. 2006), 36-46.

16. Ware, C. and Osborne, S. 1990. Exploration and virtual camera control in virtual three dimensional environments. SIGGRAPH Comput. Graph. 24, 2 (Mar. 1990), 175-183.

17. Wigdor, D., Shen, C., Forlines, C., and Balakrishnan, R. 2006. Effects of display position and control space orientation on user preference and performance. In Proceedings of the SIGCHI Conference on Human Factors in Computing Systems (Montréal, Québec, Canada, April 22 - 27, 2006). R. Grinter, T. Rodden, P. Aoki, E. Cutrell, R. Jeffries, and G. Olson, Eds. CHI '06. ACM, New York, NY, 309-318.

18. Wigdor, D., Shen, C., Forlines, C., and Balakrishnan, R. 2006. Table-centric interactive spaces for real-time collaboration. In Proceedings of the Working Conference on Advanced Visual interfaces (Venezia, Italy, May 23 - 26, 2006). AVI '06. ACM, New York, NY, 103-107.

19.Zhai, S. 1998. User performance in relation to 3D input device design. SIGGRAPH Comput. Graph. 32, 4 (Nov. 1998), 50-54. 